Article

\title{
Aorta Ascending Aneurysm Analysis Using CFD Models towards Possible Anomalies
}

\author{
Mariana Simão ${ }^{1, *}$, Jorge M. Ferreira ${ }^{2}$, António C. Tomás ${ }^{3}$, José Fragata ${ }^{4}$ and Helena M. Ramos ${ }^{5}$ \\ 1 Department of Civil Engineering, Architecture and Georesources (DECivil), Instituto Superior Técnico, \\ Universidade de Lisboa, 1600-276 Lisboa, Portugal \\ 2 Hospital Sta Maria, Faculty of Medicine, Universidade de Lisboa, 1649-028 Lisboa, Portugal; \\ jorgemiguelferreira.19@gmail.com \\ 3 Cardiothoracic Surgery Department, Santa Marta Hospital, 1169-024 Lisbon, Portugal; \\ acruztomas@gmail.com \\ 4 Nova Medical School, chronic diseases research center (CEDOC), 1169-056 Lisbon, Portugal; \\ jigfragata@gmail.com \\ 5 Department of Civil Engineering, Architecture and Georesources (DECivil), Instituto Superior Técnico, \\ Universidade de Lisboa, 1600-276 Lisboa, Portugal; helena.ramos@tecnico.ulisboa.pt \\ * Correspondence: m.c.madeira.simao@tecnico.ulisboa.pt; Tel.: +351-218-418-151
}

Received: 14 March 2017; Accepted: 8 June 2017; Published: 10 June 2017

\begin{abstract}
Computational fluid dynamics (CFD) can be seen as complementary tool alongside the visualization capabilities of cardiovascular magnetic resonance (CMR) and computed tomography (CT) imaging for decision-making. In this research CT images of three cases (i.e., a healthy heart pilot project and two patients with complex aortic disease) are used to validate and analyse the corresponding computational results. Three 3D domains of the thoracic aorta were tested under hemodynamic conditions. Under normal conditions, the flow inside the thoracic aorta is more streamlined. In the presence of ascending aortic aneurysm, large areas of blue separation zones (i.e., low velocities) are identified, as well as an internal geometry deformation of the aortic wall, respectively. This flow separation is characterized by the reversal of flow and sudden drop of the wall shear stress (WSS) in the aorta. Moreover, the aortic aneurysm simulations adversely affect the flow by increasing the pressure drop and flow inefficiency, due to the anatomical configuration of the ascending aorta. Altered hemodynamics led to a vortex formation and locally reversed the flow that eventually induced a low flow velocity and oscillating WSS in the thoracic aorta. Significant changes in the hemodynamic characteristics affect the normal blood circulation with strong turbulence occurrence, damaging the aortic wall, leading ultimately to the need of surgical intervention to avoid fatal events.
\end{abstract}

Keywords: aortic aneurysm; computational fluid dynamic (CFD); hemodynamic analysis; wall shear stress (WSS); cardiovascular magnetic resonance (CMR); computed tomography (CT)

\section{Introduction}

According to anatomical regions, the thoracic aorta goes from the heart to the diaphragm where it enters the abdominal region, becoming the abdominal aorta, which runs until the aortic bifurcation. The aorta begins as the ascending aorta, turns into the aortic arch and takes an inferior course where the descending aorta starts its vertical trajectory before ending at the bifurcation of the two common iliac arteries (Figure 1). The ascending aorta originates in the upper part of the left ventricle, from which is separated by the aortic valve. This area, designated as the aortic root, does not have the classical tubular shape yet, but follows a bulb-shaped geometry [1]. This region is quite important as its particular shape allows the blood that recoils to be collected and be directed to the coronary ostia. The tubular 
shape, typical of the aorta, begins at the sinotubular junction, which marks the transition between the root and the ascending aorta. The ascending aorta terminates on the aortic arch, which has a steep curvature from where the supra-aortic vessels originate superiorly. It continues its course running downwards to the diaphragm, being called the descending aorta [2].

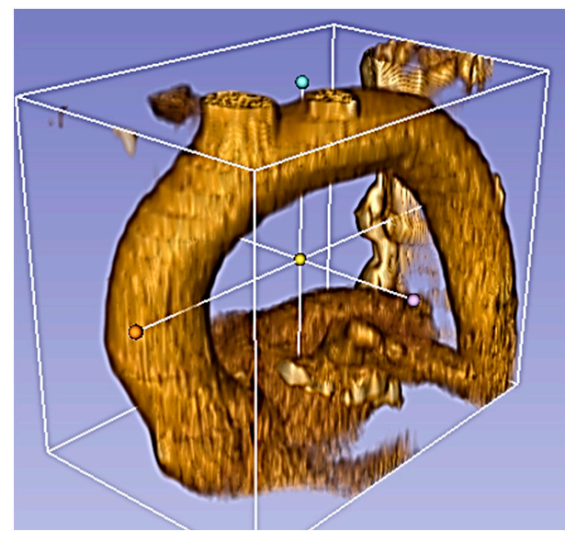

Figure 1. Physiological aorta root in the normal condition.

The ascending aorta aneurysm is the most common form of thoracic aortic aneurysm (TAA) $(60 \%)$, followed by the aneurysm of the descending aorta $(30 \%)$ and the aortic arch $(10 \%)$, while the remaining $10 \%$ are thoracic-abdominal aneurysms [3]. The TAA is defined as a dilatation of the ascending portion of the aorta that affects the segment between the aortic valve and the brachiocephalic trunk, producing an enlarged vessel diameter 1.5 times greater than the original diameter (Figure 2) [4-6]. An ascending aorta, with a diameter between 1.1 and 1.5 times that of the original size, is considered to be dilated. The typical progression of an aneurysm in the aorta begins with high blood pressure, which increases the pressure waves of blood against the walls of the aorta. The presence of atherosclerotic disease also causes the wall of the aorta to lose its elasticity which means that its ability to dilate and recoil with each pressure wave of blood induced by the heartbeat is decreased. Eventually, the elastic membranes and smooth muscle within the aortic wall deteriorate and the vessel diameter increases [7].

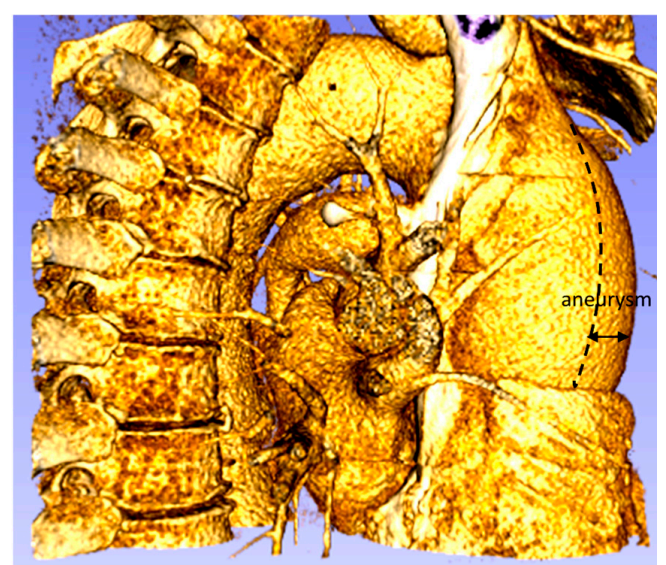

(a)

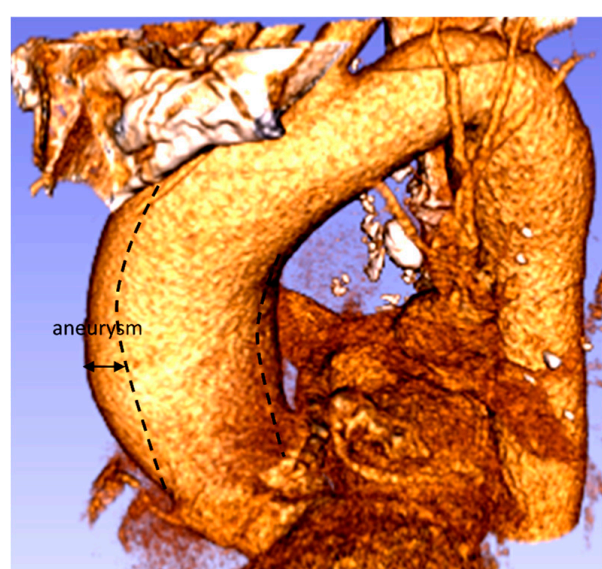

(b)

Figure 2. Aneurysm of the ascending thoracic aorta: (a) Patient 1; (b) Patient 2.

Understanding the physiology of patients with aorta disease is crucial not only for understanding local hemodynamics in complex anatomies, but also refining the assessment of individual patients [8]. Cardiovascular magnetic resonance (CMR) and computed tomography (CT) imaging have been very useful in evaluating blood flow characteristics [9-11]. Although this imaging technique (i.e., CMR) 
provides a 3D flow map of the blood circulation and can be used to explain complex hemodynamic scenarios, simulating multiple conditions for the same patient is still relatively long (15 $\mathrm{min}$ ). Additionally, with limited spatial resolution compared to CT, despite the efforts in accelerating $4 \mathrm{D}$ sequences [12], not all clinical centres are proficient in using this technique [8]. Thus, CFD analysis reveals to be a relevant potential clinical tool to predict mal functions or future anomalies.

Computational fluid dynamics are capable to predict different scenarios at a patient-specific level [13]. Nevertheless, for computational models to be potentially integrated into clinical practice or used as clinical data (i.e., for decision-making) they need to be validated. Thus, in the present research two computational models were developed: one representative of a complex aortic pathology, with the presence of aneurysms, and another illustrative of a normal heart condition.

\section{Materials and Methods}

\subsection{Patient Data}

Patients with aortic pathology underwent CT evaluation of cardiac anatomy and function. The computed axial tomography (CT), with a spatial resolution of $0.5-0.625 \mathrm{~mm}$ in the $z$-axis, and approximately $0.5 \mathrm{~mm}$ in the $x$ to $y$-axes, showed that, morphologically, two of the patients presented aneurysms in the thoracic artery trunk. Figures 3-5 show the CT derived anatomical data.
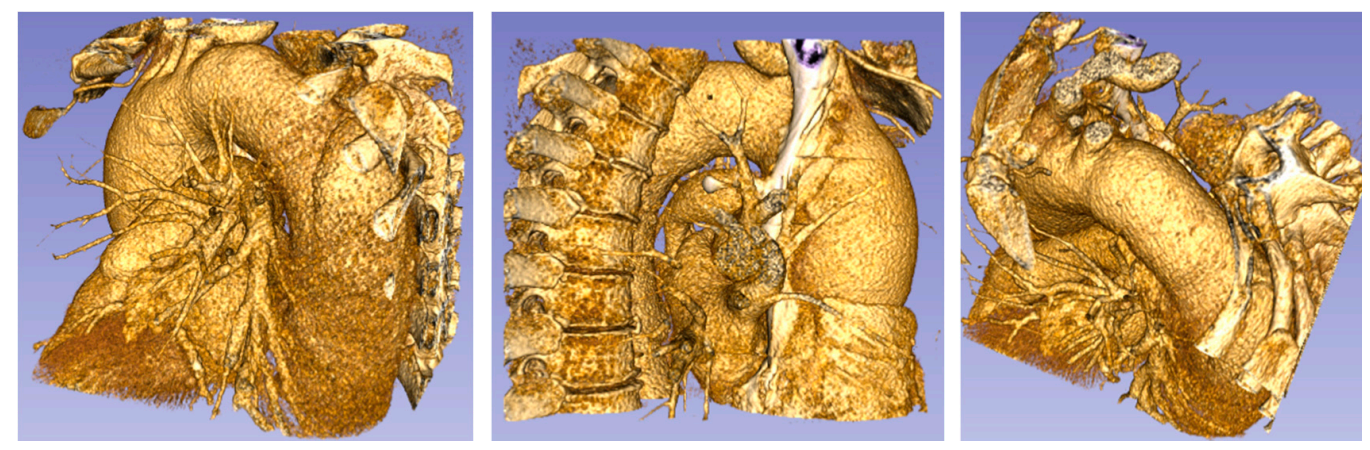

Figure 3. Patient 1 (73 years old) with a thoracic aorta aneurysm.
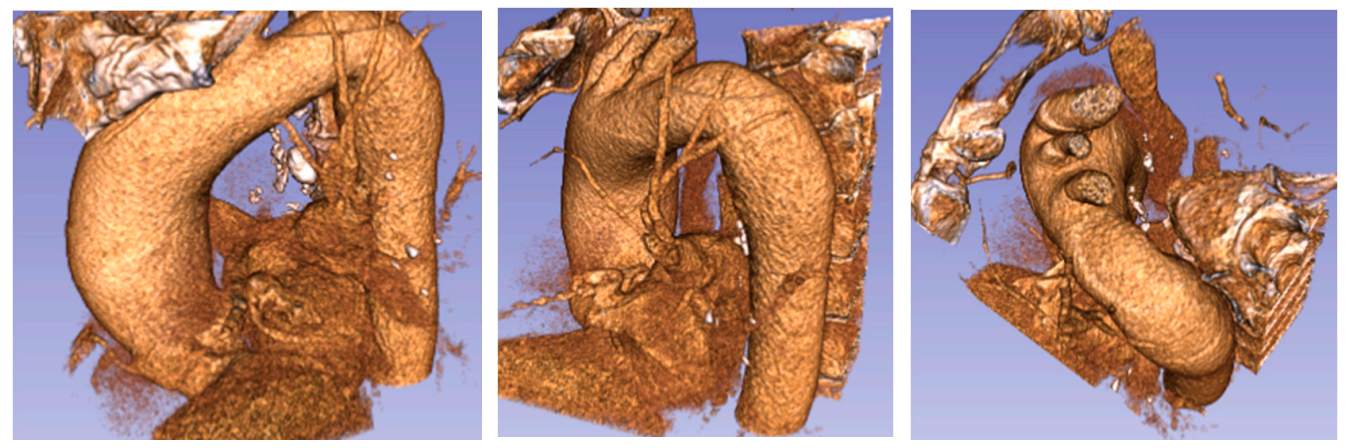

Figure 4. Patient 2 (76 years old) with an ascending aorta aneurysm.

Figure 5 shows the aortic root with the aortic diameter almost constant along the entire thoracic course. 

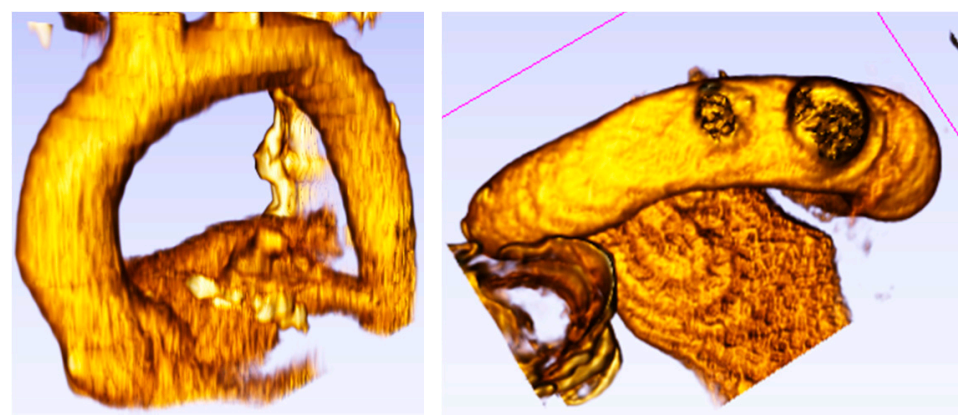

Figure 5. Healthy patient (pilot) (62 years old) without aorta disease.

\subsection{Model Geometry and Mesh}

The anatomic model was derived from the CT scan of the three patients. 3D volumes of the thoracic aorta were generated using the commercial software COMSOL Multiphysics 4.3.b (COMSOL Inc., Burlington, MA, USA). The hemodynamic analysis included four steps: (i) the aorta vessel geometry of the patients; (ii) the 3D image data in digital imaging and communications in medicine (DICOM) format was reconstructed into 3D anatomic geometry using the medical open source imaging software 3D Slicer 4.6.2.; (iii) the 3D patient-specific geometries were extracted in the numerical domain (stereolithography (STL) format); and (iv) the numerical domain was meshed and simulations were performed on the CFD COMSOL-Multiphysics 4.3.b model. To verify the specific differences of aorta pathologies (Figures 6 and 7) compared to the typical arrangement, an aortic root from a healthy patient (i.e., without aortic pathology) was also generated (Figure 8).

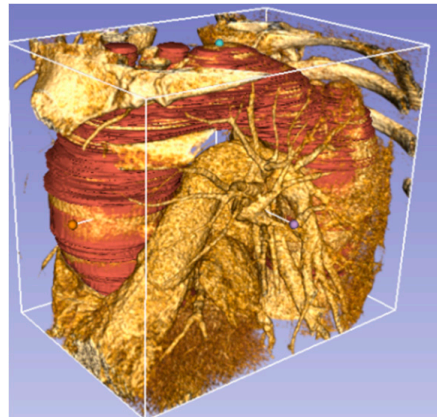

(a)

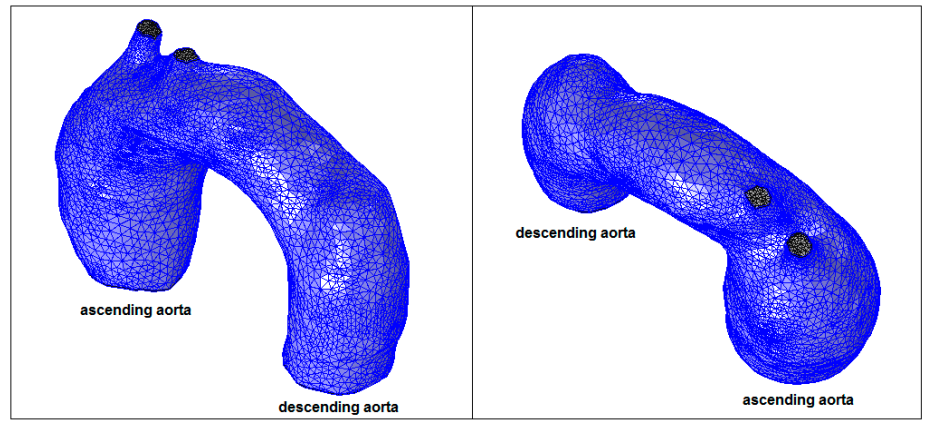

(b)

Figure 6. Ascending aorta aneurysm (Patient 1): (a) computed tomography (CT) data and segmentation; and (b) mesh.

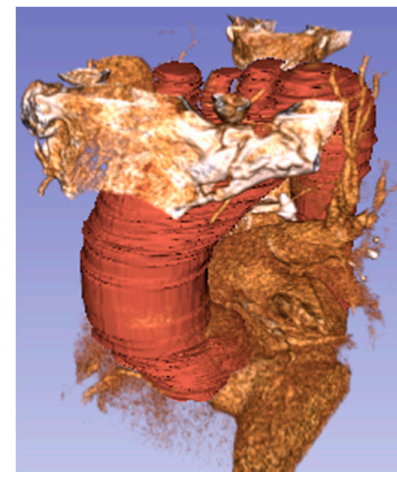

(a)

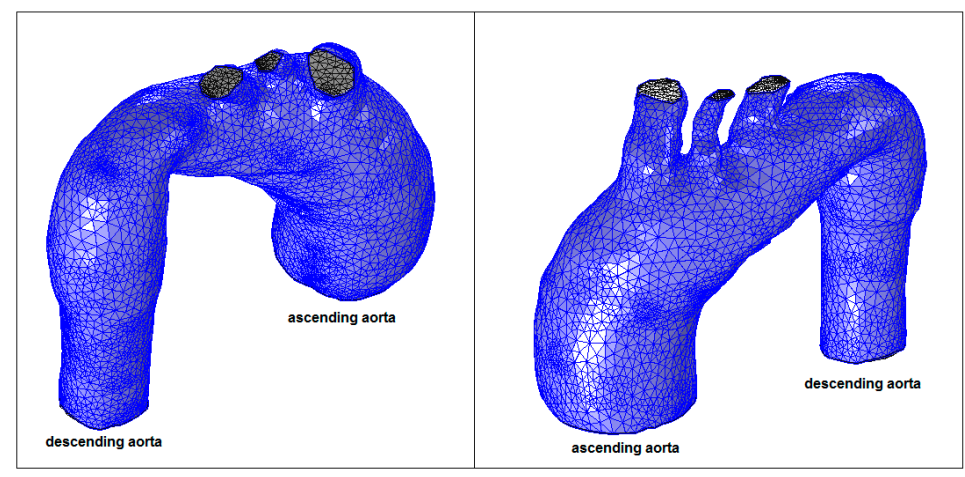

(b)

Figure 7. Ascending aorta aneurysm (Patient 2): (a) CT data and segmentation; and (b) mesh. 


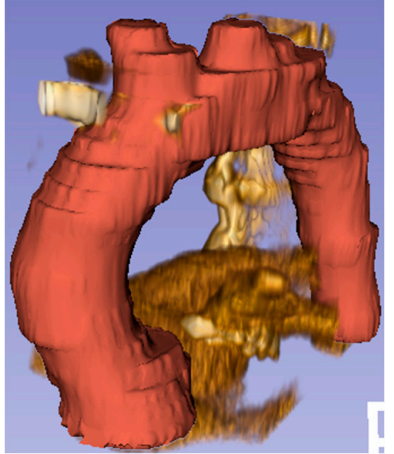

(a)

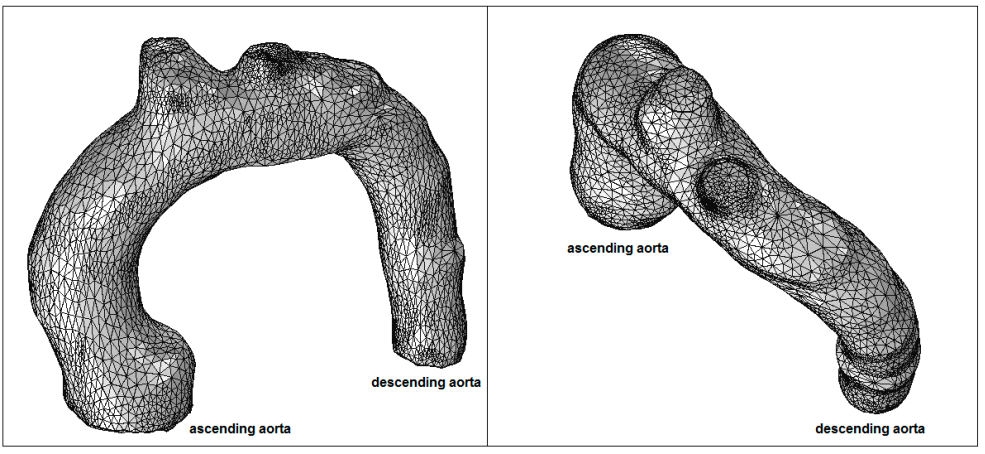

(b)

Figure 8. Healthy patient (pilot): (a) CT data and segmentation; and (b) mesh.

The provided STL geometry presents some relatively complex surfaces, fillets, and blends, which result in small faces in some parts of the model. These small faces force the tet-mesher (tetrahedral mesh for 3D models) to use smaller elements. A solution is to use the COMSOL tetrahedral meshing algorithm that first applies a mesh on all of the surfaces of an object. This mesh is then used to seed the volume mesh from which tetrahedral elements grow elements inwards. As these tetrahedral elements intersect, their sizes are adjusted with the objective of keeping the elements as isotropic (similar edge lengths and included angles) as possible and to have reasonably gradual transitions between smaller and larger elements [14]. However, when these algorithms attempt to remove topological entities, they often need to modify the underlying NURBS (non-uniform rational basis spline) surfaces and are, therefore, somewhat limited. An alternative in COMSOL Multiphysics software is to use Virtual Operations, which can keep the existing geometrical representations as a basis for constructing a new alternative topological structure purely for the purposes of meshing and defining the physics [14].

From the Virtual Operations options, the Form Composite Faces feature was selected resulting in the same geometry, but ignoring faces that lie between two adjacent domains, resulting in a single domain. Lastly, the Mesh Control Points, Edges, Faces, and Domains features, hide points, edges, faces, or domains during the set-up of the physics. However, these geometric entities will still be present during the meshing step. By using these types of operations during the computational mesh of the anatomical models, greater control over the meshing process is done by designating geometric entities for the control of the mesh size and distribution [14].

Then, to assess the performance of each structure, two kinds of analyses were undertaken: mesh convergence and mesh sensitivity analysis. These two analyses are both based on the concept of establishing the independence of the CFD results over the mesh quality. A mesh sensitive analysis was developed by refining the mesh until a critical result, such as the pressure, converges (i.e., does not change significantly with each refinement) [15]. As shown in Figure 9 when the mesh number increases to 200,000, the pressure at the condition of peak systolic converges to a constant value. Thus, a mesh with 225,059 elements for a normal aorta and 2,358,055 and 242,859 elements, respectively, for Patient 1 and 2, were used in this research.

The convergence analysis was done beginning with a very coarse mesh and incrementing the smoothness of the mesh by $25 \%$ for each further step, reaching $150 \%$ of the augmented mesh density [16]. 


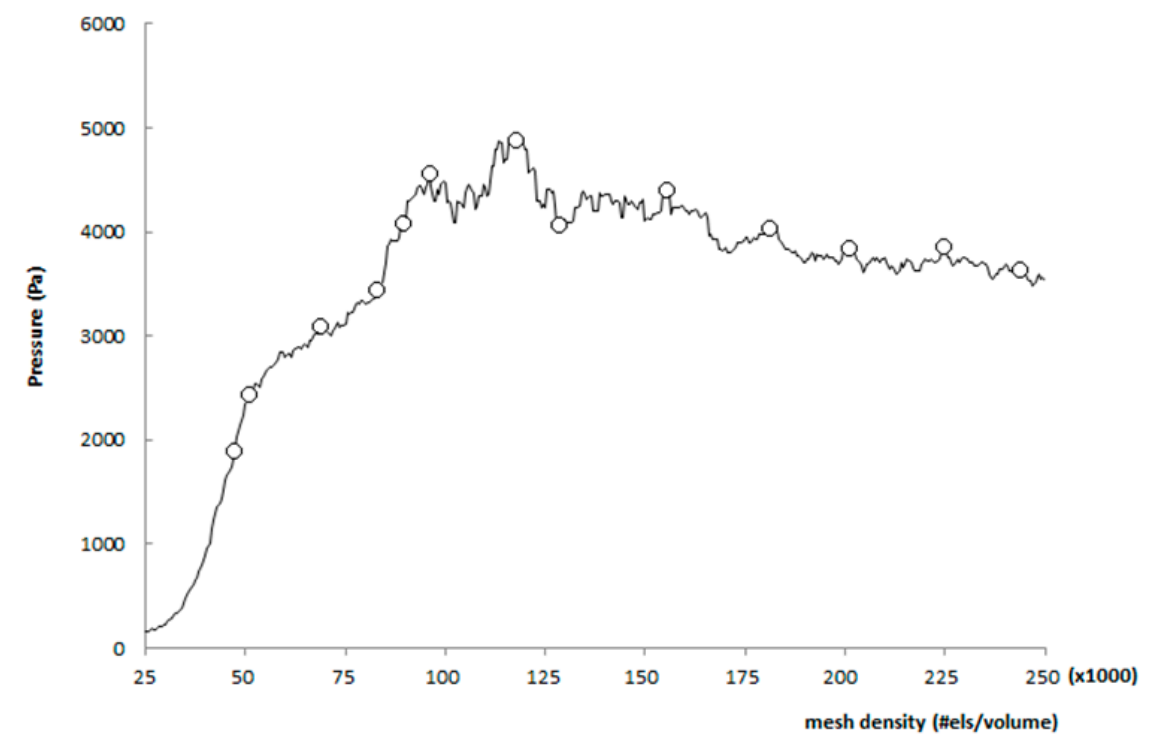

Figure 9. Mesh sensitivity analysis.

\subsection{Boundary Conditions}

The FEM involves dividing the problem into subdomains, each represented by a set of element equations to the original problem, followed by systematically recombining all sets of element equations into a global system of equations for the final calculation. The global system of equations has known solution techniques and can be calculated from the initial values of the original problem to obtain a numerical answer [7]. The 3D incompressible Navier-Stokes equations were used for blood flow simulations, with the CFD software COMSOL-Multiphysics Equation (1):

$$
\begin{gathered}
\rho \frac{\partial v}{\partial t}+\rho(v \cdot \nabla) v-\nabla \sigma=0 \quad \text { in } \Omega^{t}, \quad \forall t \in I \\
\nabla \cdot v=0 \quad \text { in } \Omega^{t}, \quad \forall t \in I
\end{gathered}
$$

where $I=[0, T]$ is the time interval and $\rho$ is the density of blood. The unknown variables are the blood velocity, $v$, and the pressure that depends on the Cauchy stress, $\sigma(v, p)$, described by a constitutive relation that characterizes the rheology of the fluid. In the case of Newtonian fluids the total stress tensor is simply defined by $\sigma(v, p)=-P I+2 \mu D(v), \mu$ is the dynamic viscosity of blood, and $D$ is the strain rate tensor, given by $D(v)=\left(\nabla v+\nabla v^{T}\right) / 2$. In this research, the viscosity is variable and depends on the shear rate [17]:

$$
\gamma=\sqrt{2 \operatorname{tr}\left(D^{2}\right)}
$$

Thus, for a non-Newtonian fluid, the total stress tensor is given by:

$$
\sigma(v, p)=-P I+2 \mu(\dot{\gamma}) D(v)
$$

Different non-Newtonian models have been proposed in expert literature, describing different specifications of the viscosity function $\mu(\gamma)$. In case of blood flows the focus will fall on the bounded viscosity laws, using Carreu's shear-thinning model Equation (4) [17]:

$$
\mu=\mu_{\infty}+\left(\mu_{0}-\mu_{\infty}\right)\left(1+(\lambda \gamma)^{a}\right)^{\frac{n-1}{a}}
$$


where $\gamma$ is the rate of the deformation tensor given by Equation (5) for a 3D Cartesian coordinate system [18]:

$$
\gamma=\left[2\left\{\left(\frac{\partial v_{x}}{\partial x}\right)^{2}+\left(\frac{\partial v_{y}}{\partial y}\right)^{2}+\left(\frac{\partial v_{z}}{\partial z}\right)^{2}\right\}+\left(\frac{\partial v_{x}}{\partial y}+\frac{\partial v_{y}}{\partial x}\right)^{2}+\left(\frac{\partial v_{x}}{\partial z}+\frac{\partial v_{z}}{\partial x}\right)^{2}+\left(\frac{\partial v_{y}}{\partial z}+\frac{\partial v_{z}}{\partial y}\right)^{2}\right]^{1 / 2}
$$

The constants for the Carreau model were established from in vivo data according to [17]:

$$
\begin{array}{cc}
\mu_{0}=0.042 \text { Pa.s } & \lambda=3.31 s \\
\mu_{\infty}=0.00345 \text { Pa.s } & n=0.375
\end{array}
$$

in which the coefficients $\mu_{0}$ and $\mu_{\infty}$ are the asymptotic viscosities, that depend on the lowest shear rates, which corresponds to the lowest pressure drop in a pipe flow and on the higher shear rates, respectively. Herein, $a=2$, for the Carreu model and $\lambda$ and $n$ are parameters to be estimated. If $\lambda=0$ or $n=1$, the viscosity is constant and the Newtonian model is recovered with the viscosity $\mu_{0}$. These parameters represent the relaxation time $(\lambda)$, i.e., they determine the value $\gamma$ at which the low shear rate ends [19]. The power law index $(\mathrm{n})$ represents the slope of the rapidly-decreasing portion of the curve and the shape parameter (a) is the shape transition between the upper $\gamma$ and the rapidly-increasing portion of the curve (i.e., estimated by curve fitting of experimental data, see [20-22].

A time-varying flow rate was imposed at the inlet boundary condition. As for the outlet condition, a pressure wave was set (both adapted from [23]). The boundary conditions are defined in Figure 10.

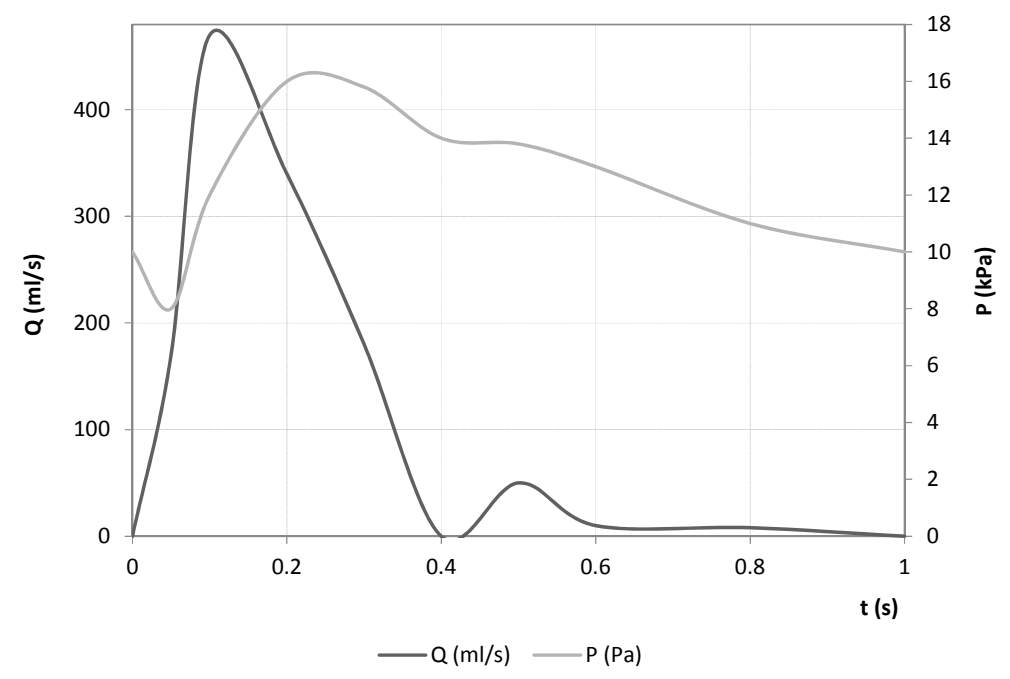

Figure 10. Boundary conditions used in both models.

The simulations used for WSS assessment typically assume that the arterial walls are rigid, which greatly simplifies the simulation process. The effect of wall motion on velocity and WSS fields is often assumed to be negligible. This may be reasonable in older subjects and certain patient groups with increased vascular stiffness. Thus, for simplicity, and also due to the aging of the patients, the vessel walls were assumed to be rigid and the no-slip condition was considered.

\section{Results}

\subsection{Blood Velocity}

Figure 11 shows normal streamlines velocities in the aorta system (between $0.1 \mathrm{~m} / \mathrm{s}$ and $0.55 \mathrm{~m} / \mathrm{s}$ ) with the absence of vortex formation in the arch branches. 


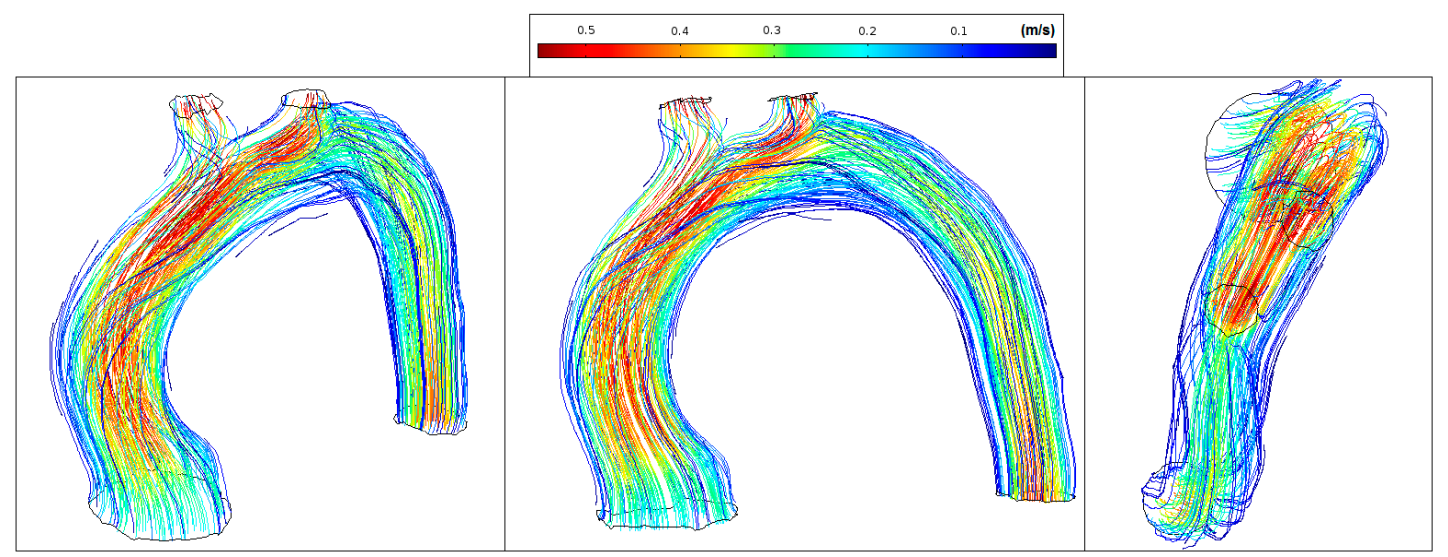

Figure 11. Healthy patient: velocity streamlines (peak systole).

Figure 12 shows low velocity values in the aorta system $(<0.35 \mathrm{~m} / \mathrm{s})$ and marked vortex formation located at the proximal segment of the artery (coded blue).

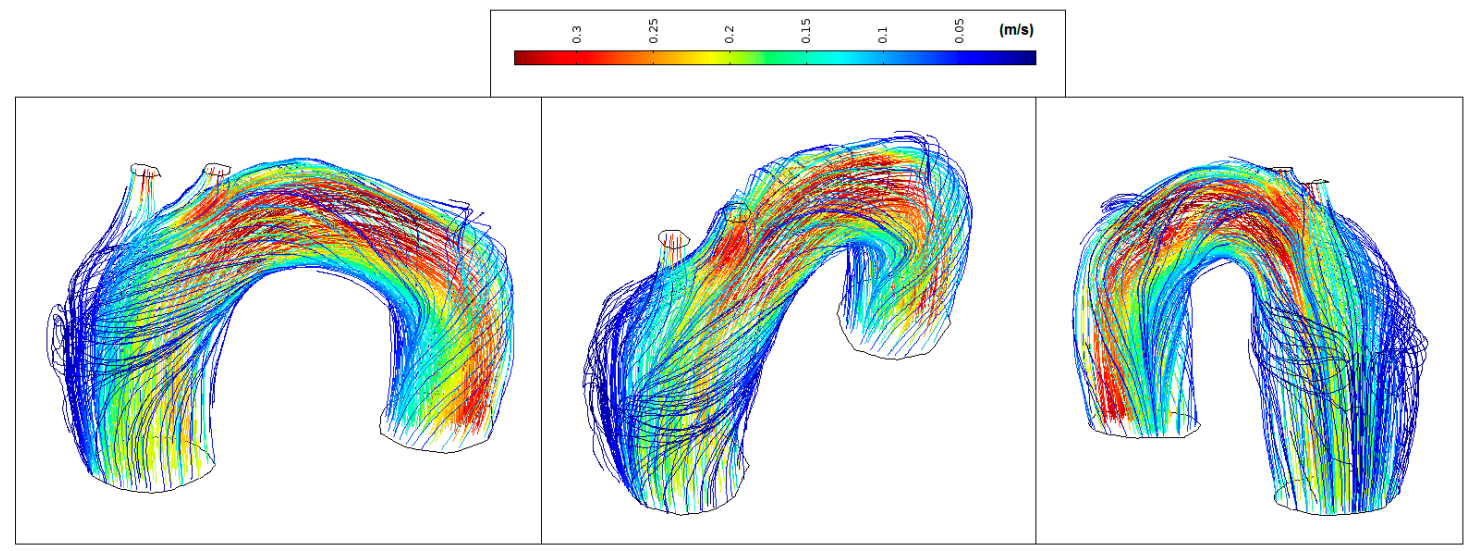

Figure 12. Patient 1: velocity streamlines (peak systole).

Figure 13 shows low velocity values in the ascending aorta $(<0.2 \mathrm{~m} / \mathrm{s})$ and marked vortex formation located at the beginning of the descending aorta (coded blue). The transition degree between the distal arch and proximal descending aorta changes abruptly making almost 90 degrees between them. This results in disturbed flow and sudden flow acceleration (coded red).

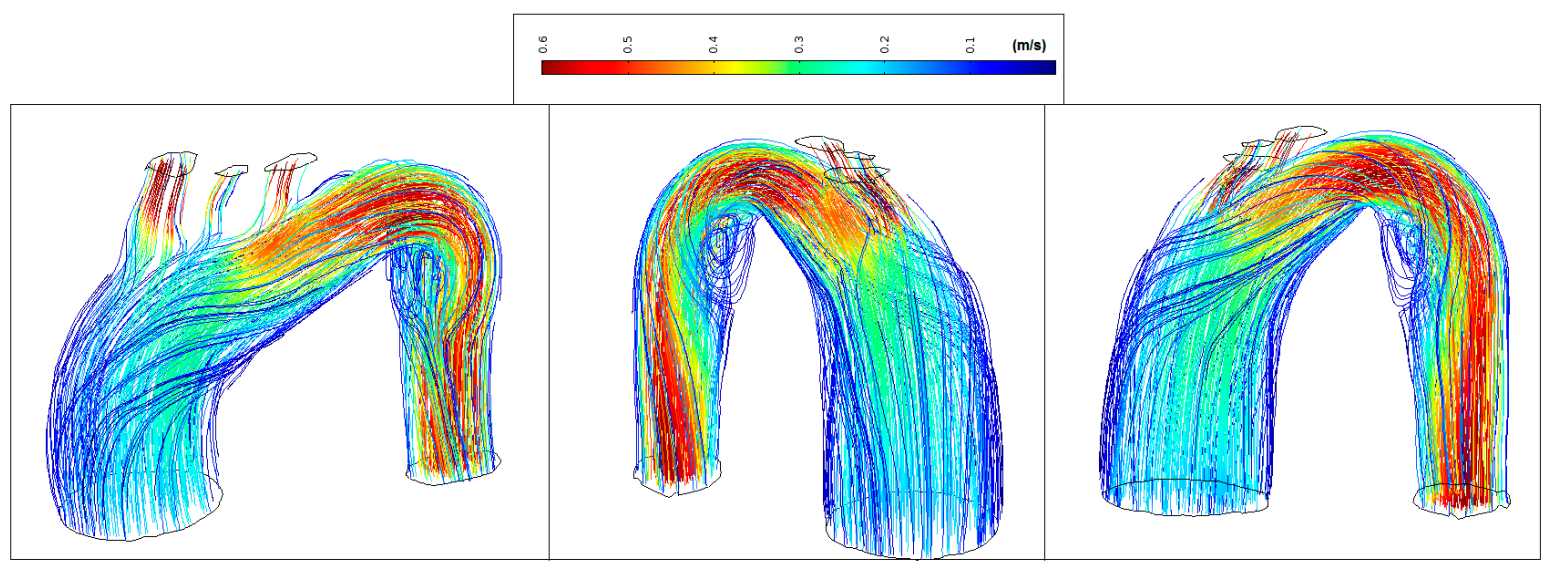

Figure 13. Patient 2: velocity streamlines (peak systole). 


\subsection{Wall Shear Stress}

It is well recognized that a higher or lower WSS (HWSS and LWSS) may cause intimal dysfunction, which could result in the progression of atherosclerosis [24]. Extremely high values of WSS are associated with vessel remodelling responsible for aneurysm initiation and progression $[25,26]$. Conversely, regions of low WSS correlate with inward vascular remodelling and the progression of atherosclerosis [27]. Altered hemodynamics led to a vortex formation and locally reverses the flow, which eventually induces low and oscillating WSS in the thoracic aorta. In comparison with the smooth aorta artery (patient-pilot, Figure 14), low WSS values in Patients 1 and 2 can be observed in the ascending thoracic aorta (Figures 15 and 16).

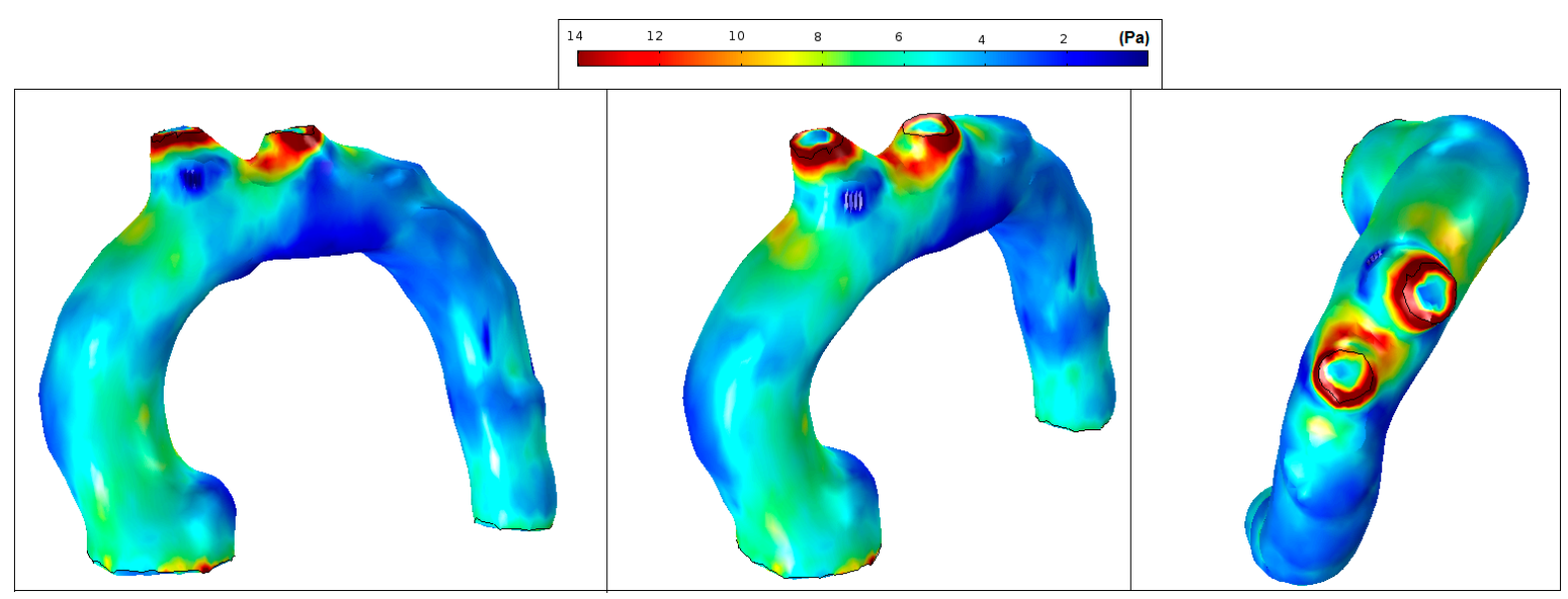

Figure 14. Patient-pilot: wall shear stress (WSS) contours (at peak systole).

Figure 14 shows normal values of WSS located in the smooth curvature of the aorta artery. On the contrary, Patients 1 and 2 present vascular areas exposed to low WSS over time, which contributes to a vascular remodelling. Herein, there is direct evidence of an aneurysm, since locally-altered hemodynamics and vessel wall parameters, i.e., the observed overt difference in values between the anterior and the posterior vessel wall, affect the vascular remodelling and, consequently, the aneurysm growth [27]. Figure 15 shows regions of low WSS correlated with the progression of atherosclerosis. Altered hemodynamics led to a vortex formation and locally reverse the flow, which induces low and oscillating WSS in the thoracic aorta. Figure 16 presents low WSS values at the inner curvature of the thoracic aorta and high WSS at the level of the aorta arch, which may explain the progression/extension of the disease in the future.

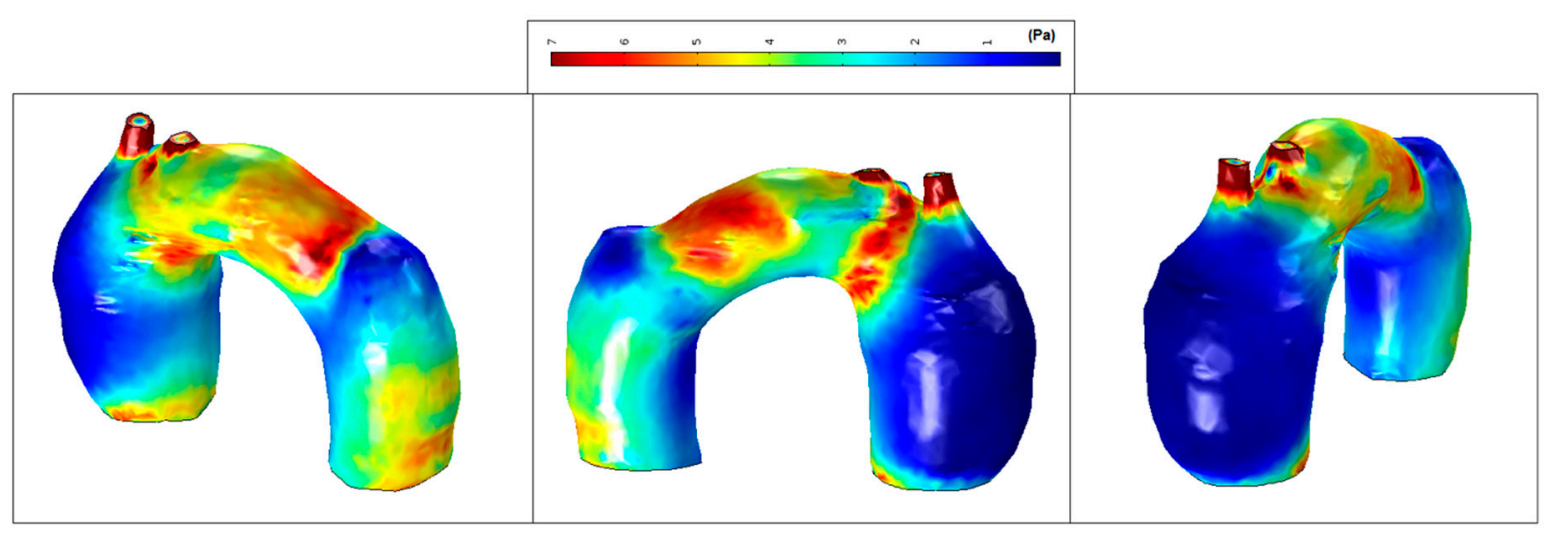

Figure 15. Patient 1: wall shear stress (WSS) contours (at peak systole). 


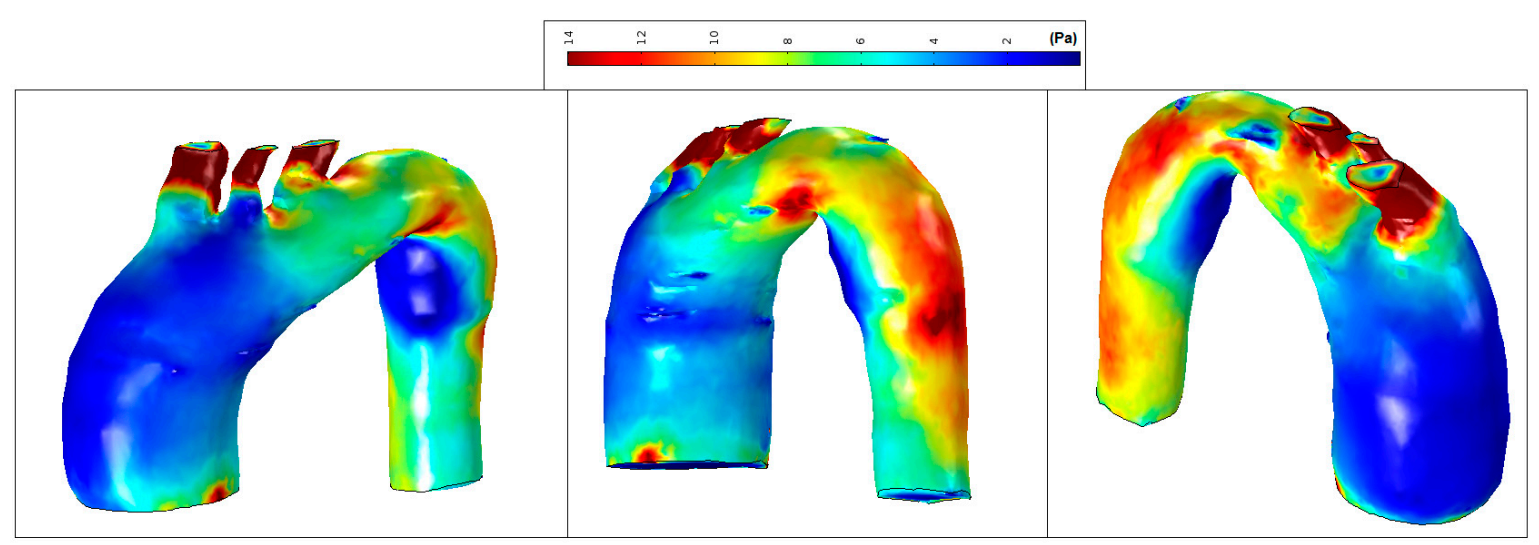

Figure 16. Patient 2: wall shear stress (WSS) contours (at peak systole).

\section{Discussion}

The computational simulations concerned the profiles of velocity and WSS in the three cases. According to [2], to characterize the structure of the TAA, the main geometrical TAA parameters are measured: $L_{\mathrm{TAA}}$, the aneurysm length, and $D_{\mathrm{TAA}}$, the maximum diameter of the aneurysm. The factor which assesses the length $L_{\mathrm{TAA}}$ and the diameter $D_{\mathrm{TAA}}$ is known as the saccular index $(\gamma)$ Equation (7) [28]. If the saccular index is close to 1, the aneurysm is saccular (spherical), but if it is close to 0 , the aneurysm is more fusiform. The deformation diameter rate $\chi$ characterizes the nondeformed aorta diameter $\left(d_{\text {anterior }}\right)$ with the maximum diameter of the aneurysm. A nonaneurysmal aorta is defined as $D_{\mathrm{TAA}}=d_{\text {anterior }}[2]$ :

$$
\begin{gathered}
\beta=\frac{r}{R} \\
\gamma=\frac{D_{\mathrm{TAA}}}{L_{\mathrm{TAA}}} \\
\chi=\frac{D_{\mathrm{TAA}}}{d_{\text {anterior }}}
\end{gathered}
$$

To evaluate the asymmetry $\beta$ of the aneurysm, $r$ and $R$ are defined as the ratio measured at the midsection of the TAA from the longitudinal $z$-axis to the posterior and anterior walls, respectively [29]. Thus, $\beta=1$ yields an azimuthal symmetry and $\beta=0.2$ is a TAA for which only the anterior wall is dilated, while the posterior wall is nearly flat [30]. Based on these indices, it is possible to identify the type of aneurysm presented in each patient (Table 1).

Table 1. Geometrical characteristics of the aneurysms.

\begin{tabular}{cccccccccc}
\hline Patient & $\begin{array}{c}d_{\text {anterior }} \\
(\mathbf{c m})\end{array}$ & $\boldsymbol{r ( \mathbf { c m } )}$ & $\boldsymbol{R}(\mathbf{c m})$ & $\begin{array}{c}\boldsymbol{D}_{\text {TAA }} \\
(\mathbf{c m})\end{array}$ & $\begin{array}{c}\boldsymbol{L}_{\text {TAA }} \\
(\mathbf{c m})\end{array}$ & $\chi$ & $\beta$ & $\gamma$ & Type \\
\hline Pilot & 2.80 & 1.40 & 1.40 & 2.80 & - & 1.00 & 1.00 & - & $\begin{array}{c}\text { saccular with azimuthal } \\
1\end{array}$ \\
3.54 & 2.06 & 2.70 & 4.76 & 6.92 & 1.35 & 0.76 & 0.69 & $\begin{array}{c}\text { symmetry (35\% of deformation) } \\
\text { fusiform with only anterior wall } \\
\text { dilated (44\% of deformation) }\end{array}$ \\
\hline
\end{tabular}

According to [1,31-35], based on the TAA shape, different criteria for the TAA collapsibility can be applied: (i) if the TAA diameter is $45 \%$ higher than the non-deformed aorta diameter; (ii) if the asymmetry index factor is $\beta<0.4$; (iii) if the deformation diameter ratio $\chi>3 \times d_{\text {anterior; }}$ and (iv) if the saccular index $\gamma<0.6$. However, the TAA dilation and potential rupture also depend on the hemodynamic loads. Thus, alternative methods of TAA rupture assessment are needed, such as numerical analysis involving CFD models to determine the WSS distributions and flow patterns (Table 2). 
Table 2. Velocity magnitude and WSS ranges.

\begin{tabular}{ccc}
\hline Patients & Velocity Magnitude Range $\mathbf{( m / s )}$ & WSS Range (Pa) \\
\hline Patient-pilot & $0.1-0.55$ & $2-14$ \\
& & $4<50 \%$ area $<6$ (LWSS) \\
Patient 1-TAA & $0.1-0.35$ & $8<50 \%$ area $<12$ (HWSS) \\
& & $1-7$ \\
Patient 2-TAA & $0.1-0.60$ & $0-14$ \\
& & $0<60 \%$ area $<5$ (LWSS) \\
& $8<40 \%<11$ (LWSS) \\
\hline
\end{tabular}

The mean WSS was set according to the values obtained for patient-pilot, i.e., 5.4 Pa. The areas with WSS values under the mean value have been classified as low WSS areas (blue) and areas with WSS values higher than the mean value as high WSS (brown) [36,37]. Analysis of WSS contours for all cases shows that the area of low WSS coincides with the location of the recirculation areas (low velocities), and higher WSS values are found in two regions: in the neck area and in the corresponding area where the blood flow jet has an impact on the aneurysmal sac [38-40]. Higher blood flow has an impact directly on the arterial wall producing higher WSS. WSS peak values may not influence too much in aneurysm growth; however, in these areas a material failure could be produced provoked by blood flow jet, as well as intrinsic properties of the vessel wall (e.g., collagen diseases). The blood flow jet path is provoked by the TAA tortuosity (see Figure 17, Patient 2, the transition between the aortic arch and descending aorta).

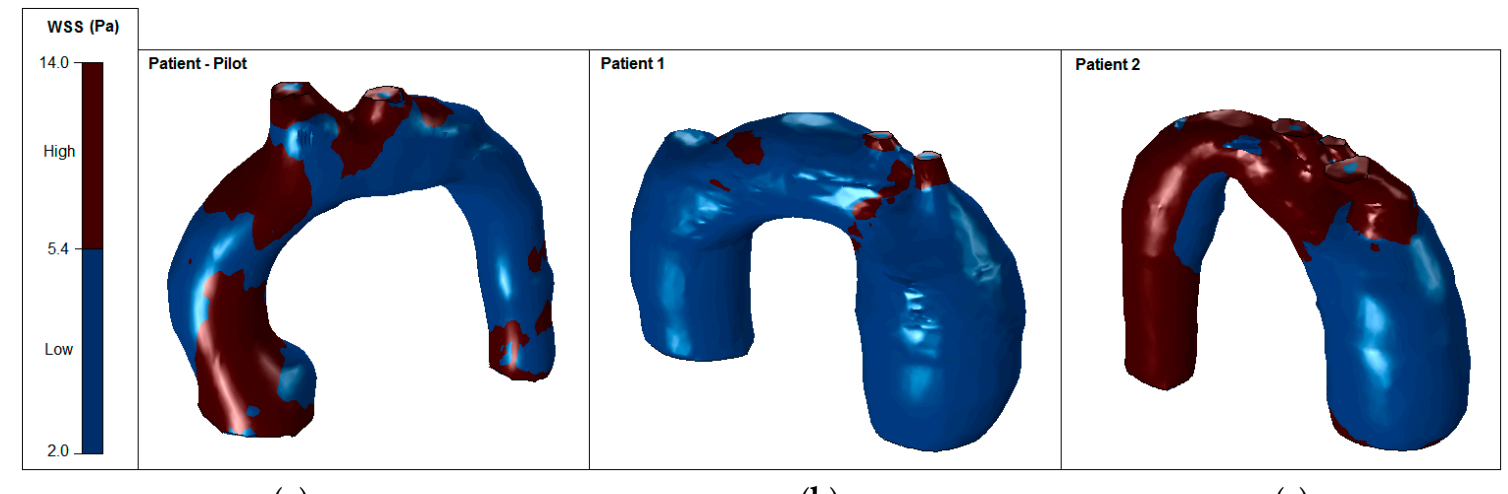

(a)

(b)

(c)

Figure 17. High and low WSS at peak systole: (a) Patient-pilot; (b) Patient 1; (c) Patient 2.

To explain such LWSS values in Patients 1 and 2, vortical structures (VS) associated with platelet formation, adhesion, and aggregation, seem to be an indicator for diseased vessels with great diameters such as the aorta and pulmonary trunk, that are more prone to create a deposition of atherosclerotic plaque or, even more relevant, mural thrombus (Figure 18). While thrombus grows at sites of LWSS, spanwise vortices give rise to streamwise vortices, i.e., the VS are triggered by unstable low-speed streaks, by stretching rather than a roll-up mechanism. Thus, blood-flow-induced activation of platelets lead to their deposition and agglomeration at the wall exposed to LWSS. 


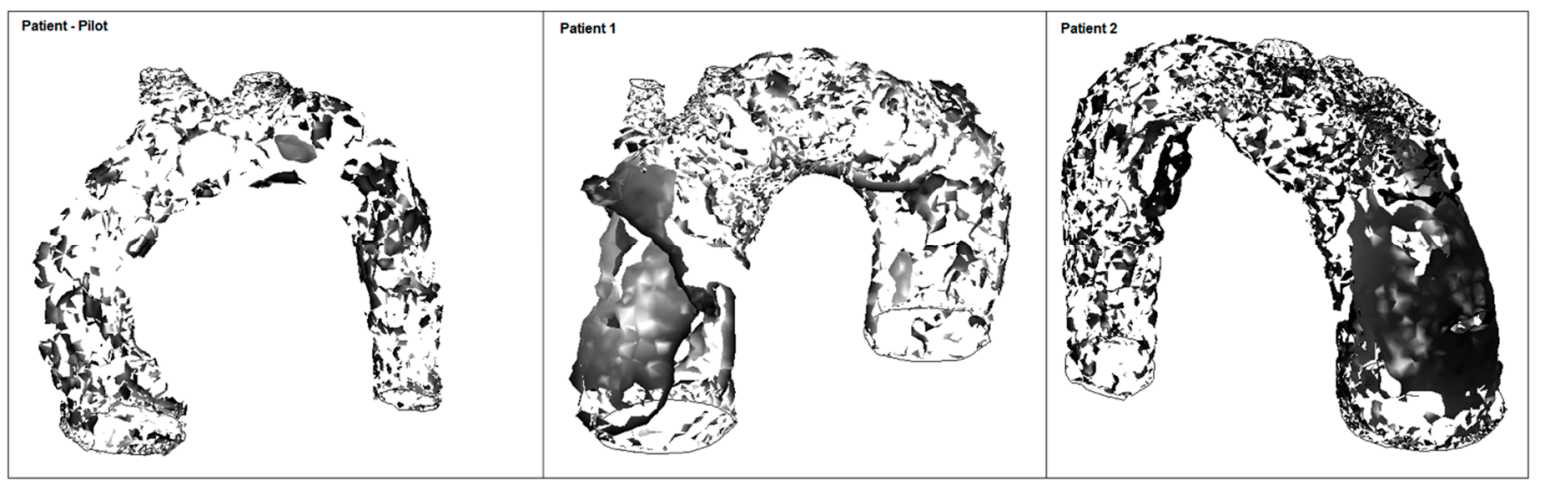

(a)

(b)

(c)

Figure 18. Formation of vortical structures (at peak systole): (a) Patient-pilot; (b) Patient 1; (c) Patient 2.

In patient-pilot, the VSs break up into smaller substructures at peak systole. In contrast to the TAA cases, streamwise VSs are reinforced at the beginning, coinciding to low WSS.

There are technical limitations regarding the validation of CFD modelling: (i) to reduce the calculation cost, a fixed boundary condition was applied in the present study, since FSI simulations require knowledge of material parameters and in vivo boundary conditions that are unknown, thus, rigid walls were assumed, neglecting the dynamics that is introduced by the moving wall; (ii) relation between aorta and other structures in the vicinity (e.g., pulsatility of the pulmonary artery); (iii) pathological changes in the endothelial tissue cannot be examined; (iv) motion constraints at initial and final boundaries, such as the compression/expansion induced by the aorta was not considered; (v) lack of in vivo data to determine if the WSS may initially have been normal and with dilation of the aorta decreased with the increasing of the diameter, or, at the beginning, when the aorta was still of normal dimensions, WSS could be higher (for some unknown reason, such as the orientation and pressure of the blood when ejected), which led to the development of the aneurysm. An FSI simulation of the model was not performed, as the subject-specific wall material properties were not available and using standard values from literature would only add uncertainty to the results.

Although this study is under research, it can contribute to a better understanding of aorta diseases and used by medical teams in optimizing specific treatment for each patient as to determine the best health procedure to follow in each case.

\section{Conclusions}

Flow characterization by definition of non-Newtonian blood flow characteristics, comparisons between levels of turbulence, WSS distribution, and vortical structures (localized streamwise vortices) were the main goals of this research, assuming a non-mobile solid border.

Vorticity streamlines in the aorta artery revealed lower wall shear stress with the progression of atherosclerosis. Platelets were exposed to longer residence time inside the vortex than in the normal aorta. The results also showed that the blood stagnation in the ascending aorta of Patients 1 and 2 are associated with low wall shear stress. By simulating the blood flow in different volumetric configurations of the systemic vasculature, using the same boundary conditions for each patient, allowed to investigate the hemodynamic quantities that are relevant to assess cardiovascular function. Analysis of WSS for all cases shows that low WSS areas coincide with the location of recirculation zones, related to low velocities, and are more pronounced in aneurysms with a saccular shape.

Analyses described in this research highlight the potential for CFD to play an important role in the clinical determination of aneurysm risks. The choice of boundary conditions, mesh and time resolution, segmentation methods and parameters, location of vessel truncation, can affect the quantitative hemodynamic results. Hence, with the use of CFD models as a complementary tool with CT imaging brings important insights to clinicians to better understand and visualize the flow 
patterns, pressure gradients, turbulence, and WSS caused by heart disease and to make predictions for possible new anomalies.

This analysis gives additional information not only for a specific case, but in the evaluation of different scenarios that can be virtually simulated: (i) geometry configuration; and (ii) evaluation of vorticities and WSS over time that cannot be identified using in vivo data.

Acknowledgments: This research was funded by the Civil Engineering Research and Innovation for Sustainability (CEris) of the Department of Civil Engineering, Architecture and Georesources, Instituto Superior Técnico (IST), University of Lisbon (ULisbon), through the research grant BL255/2016 of the first author.

Author Contributions: Mariana Simão simulated the in vivo models according to the data lent by António C. Tomás and José Fragata; all authors analysed the data; all authors contributed with materials/analysis tools; and Mariana Simão wrote the paper. Helena M. Ramos, Jorge M. Ferreira, and António C. Tomás reviewed the manuscript.

Conflicts of Interest: The authors declare no conflict of interest.

Informed Consent: Informed consent was obtained from the patient for being included in the study. All procedures followed are in accordance with the ethical standards of the responsible committee on human data and with the Helsinki Declaration of 1975, as revised in 2013.

\section{References}

1. Biasetti, J.; Hussain, F.; Gasser, T. Blood flow and coherent vortices in the normal and aneurysmatic aortas: A fluid dynamical approach to intra-luminal thrombus formation. J. R. Soc. Interface 2011. [CrossRef] [PubMed]

2. Soudah, E.; Loong, T.; Bordone, M.; Pua, U.; Narayanan, S. CFD modelling of abdominal aortic aneurysm on hemodynamic loads using a realistic geometry with CT. Comput. Math. Methods Med. 2013. [CrossRef] [PubMed]

3. Bonow, R.; Mann, D.; Zipes, D.; Libby, P. Braunwald's Heart Disease. In A Textbook of Cardiovascular Medicine; Elsevier Health Sciences: Amsterdam, The Netherlands, 2011; Volume 2.

4. Vorp, D. Biomechanics of abdominal aortic aneurysm. J. Biomech. 2007, 40, 1887-1902. [CrossRef] [PubMed]

5. Humphrey, J. Cardiovascular Solid Mechanics. In Cells, Tissues and Organs; Springer: New York, NY, USA, 2002.

6. Powell, J.; Brown, L.; Forbes, J. Final 12-year follow-up of surgery versus surveillance in the UK small aneurysm trial. Br. J. Surg. 2007, 94, 702-708. [CrossRef] [PubMed]

7. Filardi, V. CFD analysis to evaluate hemodynamic parameters in a growing abdominal aortic aneurysm. Vasc. Dis. Manag. 2015, 12, E84-E95.

8. Biglino, G.; Cosentino, D.; Steeden, J.; De Nova, L.; Castelli, M.; Ntsinjana, H.; Pennati, G.; Taylor, A.; Schievano, S. Using 4D cardiovascular magnetic resonance imaging to validate computational fluid dynamics: A case study. Front. Pediatr. 2015, 3. [CrossRef] [PubMed]

9. Valverde, I.; Nordmeyer, S.; Uribe, S.; Greil, G.; Berger, F.; Kuehne, T. Systemic-to-pulmonary collateral flow in patients with palliated univentricular heart physiology: Measurement using cardiovascular magnetic resonance 4D velocity acquisition. J. Cardiovasc. Magn. Reson. 2012, 14. [CrossRef] [PubMed]

10. Francois, C.; Srinivasan, S.; Schiebler, M.; Reeder, S.; Niespodzany, E.; Landgraf, B. 4D cardiovascular magnetic resonance velocity mapping of alterations of right heart flow patterns and main pulmonary artery hemodynamics in tetralogy of Fallot. J. Cardiovasc. Magn. Reson. 2012, 14. [CrossRef] [PubMed]

11. Geiger, J.; Markl, M.; Jung, B.; Grohmann, J.; Stiller, B.; Langer, M. 4D-MR flow analysis in patients after repair of tetralogy of Fallot. Eur. Radiol. 2011, 21, 1651-1657. [CrossRef] [PubMed]

12. Carlsson, M.; Toger, J.; Kanski, M.; Bloch, K.; Stahlberg, F.; Heiber, E. Quanification and visualization of cardiovascular $4 \mathrm{D}$ velocity mapping accelerated with parallel imaging or k-t BLAST: Head to head comparison and validation at 1.5T and 3T. J. Cardiovasc. Magn. Reson. 2011, 13. [CrossRef] [PubMed]

13. De Vecchi, A.; Nordsletten, D.; Razavi, R.; Greil, G.; Smith, N. Patient specific fluid/structure ventricular modelling for integrated cardiac care. Med. Biol. Eng. Compt. 2013, 51, 1261-1270. [CrossRef] [PubMed]

14. COMSOL. Multiphysics User's Guide; COMSOL Inc.: Burlington, MA, USA, 2012; p. 1372.

15. De Santis, G.; De Beule, M.; Van Canneyt, K.; Segers, P.; Verdonck, P.; Verhegghe, B. Full-hexahedral structured meshing for image-based computational vascular modeling. Med. Eng. Phys. 2011, 33, 1318-1325. [CrossRef] [PubMed] 
16. Baker, T.; Pebay, P.; Pousin, J. Dynamic meshing for finite element based segmentation of cardiac imagery. In Proceedings of the WCCM V-Fifth World Congress on Computational Mechanics, Vienna, Austria, 2002.

17. Sochi, T. Non-Newtonian Rheology in Blood Circulation. In Fluid Dynamics; Department of Physics and Astronomy, University College London: London, UK, 2014; Volume 1. [CrossRef]

18. Herzog, C.; Zwrner, P.; Doll, J.; Nielsen, C.; Nguyen, S.; Savino, G.; Vogl, T.; Costello, P.; Schoepf, U. Significant coronary artery stenosis: comparison on per-patient and per-vessel or per-segment basis at 64-section CT angiography. Radiology 2007, 244, 112-120. [CrossRef] [PubMed]

19. Bluestein, D.; Dumont, K.; de Beule, M. Intraluminal thrombus and risk of rupture in patient specific abdominal aortic aneurysm-FSI modelling. Comput. Methods Biomechan. Biomed. Eng. 2009, 12, $73-81$. [CrossRef]

20. Vorp, D.; Raghavan, M.; Webster, M. Mechanical wall stress in abdominal aortic aneurysm: Influence of diameter and asymmetry. J. Vasc. Surg. 1998, 27, 632-639. [CrossRef]

21. Ouriel, K.; Green, R.; Donayre, C.; Shortell, C.; Elliott, J.; DeWeese, J. An evaluation of new methods of expressing aortic aneurysm size: Relationship to rupture. J. Vasc. Surg. 1992, 15, 12-20. [CrossRef]

22. Shipkowitz, T.; Rodgers, V.G.; Frazin, L.J.; Chandran, K.B. Numerical study on the effect of secondary flow in the human aorta on local shear stresses in abdominal aortic branches. J. Biomech. 2000, 33, 717-728. [CrossRef]

23. Alastruey, J.; Xiao, N.; Fok, H.; Schaeffter, T.; Figueroa, C. On the impact of modelling assumptions in multi-sacle, subject-specific models of aortic heamodynamics. J. R. Soc. Interface 2016, 13. [CrossRef] [PubMed]

24. Jung, H.; Choi, J.; Park, C. Asymmetric flows of non-newtonian fluids in a symmetric stenosed artery. Korea-Aust. Rheol. J. 2004, 1, 101-108.

25. Migliavacca, F.; Petrini, L.; Montanari, V.; Quagliana, I.; Auricchio, F.; Dubini, G. A predictive study of the mechanical behaviour of coronary stents by computer modelling. Med. Eng. Phys. 2005, 27, 13-18. [CrossRef] [PubMed]

26. Kajzer, W.; Kackmarek, M.; Marciniak, J. Biomechanical analysis of stent-oesophagus system. J. Mater. Process. Technol. 2005, 162-163, 196-202. [CrossRef]

27. Gundert, T. Improving Cardiovascular Stent Design Using Patient-Specific Models and Shape Optimization. Master's Thesis, Marquette University, Milwaukee, WI, USA, 2011; p. 104.

28. Kleinstreuer, C.; Li, Z. Analysis and computer program for rupture-risk prediction of abdominal aortic aneurysms. Biomed. Eng. Online 2006, 5. [CrossRef] [PubMed]

29. Vorp, D.; Lee, P.; Wang, D. Association of intraluminal thrombus in abdominal aortic aneurysm with local hypoxia and wall weakening. J. Vasc. Surg. 2001, 34, 291-299. [CrossRef] [PubMed]

30. Nicholls, S.; Gardner, J.; Meissner, M.; Johans, K. Rupture in small abdominal aortic aneurysms. J. Vasc. Surg. 1998, 28, 884-888. [CrossRef]

31. Bauer, M.; Siniawski, H.; Pasic, M.; Schaumann, B.; Hetzer, R. Different hemodynamic stress of the ascending aorta wall in patients with bicuspid and tricuspid aortic valve. J. Card. Surg. 2006, 21, 21820. [CrossRef] [PubMed]

32. Morbiducci, U.; Ponzini, R.; Rizzo, G.; Cadioli, M.; Esposito, A.; Montevecchi, F.M.; Redaelli, A. Mechanistic insight into the physiological relevance of helical blood flow in the human aorta an in vivo study. Biomech. Model. Mechanobiol. 2010, 10, 339-355. [CrossRef] [PubMed]

33. Hsu, M.C.; Kamensky, D.; Bazilevs, Y.; Sacks, M.S.; Hughes, T.J. Fluid-structure interaction analysis of bioprosthetic heart valves: Significance of arterial wall deformation. Comput. Mech. 2014, 54, 10551071. [CrossRef] [PubMed]

34. Lee, C.-H.; Liu, K.S.; Jhong, G.H.; Liu, S.J.; Hsu, M.Y.; Wang, C.J.; Hung, K.C. Finite element analysis of helical flows in human aortic arch: A novel index. Biomicrofluidics 2014, 8, 024111.

35. Bonomi, D.; Vergara, C.; Faggiano, E.; Stevanella, M.; Conti, C.; Redaelli, A.; Puppini, G.; Faggian, G.; Formaggia, L.; Luciani, G.B. Influence of the aortic valve leaflets on the fluid-dynamics in aorta in presence of a normally functioning bicuspid valve. Biomechan. Model. Mechanobiol. 2015, 14, 1349-1361. [CrossRef] [PubMed]

36. Morbiducci, U.; Ponzini, R.; Rizzo, G.; Cadioli, M.; Esposito, A.; De Cobelli, F.; Del Maschio, A.; Montevecchi, F.M.; Redaelli, A. In vivo quantification of helical blood flow in human aorta by time-resolved three-dimensional cine phase contrast magnetic resonance imaging. Ann. Biomed. Eng. 2009, 37, 516-531. [CrossRef] [PubMed] 
37. Guzzetti, S.; Passerini, T.; Slawinski, J.; Villa, U.; Veneziani, A.; Sunderam, V. Platform and algorithm effects on computational fluid dynamics applications in life sciences. Future Gener. Comput. Syst. 2017, 67, 382-396. [CrossRef]

38. Suh, G.Y.; Les, A.S.; Tenforde, A.S.; Shadden, S.C.; Spilker, R.L.; Yeung, J.J.; Cheng, C.P.; Herfkens, R.J.; Dalman, R.L.; Taylor, C.A. Hemodynamic changes quantified in abdominal aortic aneurysms with increasing exercise intensity using MR exercise imaging and image-based computational fluid dynamics. Ann. Biomed. Eng. 2011, 39, 2186-2202. [CrossRef] [PubMed]

39. Bird, R.B.; Armstrong, R.C.; Hassager, O. Dynamics of Polymeric Liquids, Vol 1: Fluid Mechanics, 2nd ed.; Wiley: Hoboken, NJ, USA, 1987; ISBN 978-0-471-80245-7.

40. Carreau, P.J.; De Kee, D.C.R.; Chhabra, R.P. Rheology of Polymeric Systems, Principles and Applications; Hanser/Gardner Publications: Cincinnati, OH, USA, 1997; p. 520.

2017 by the authors. Licensee MDPI, Basel, Switzerland. This article is an open access article distributed under the terms and conditions of the Creative Commons Attribution (CC BY) license (http://creativecommons.org/licenses/by/4.0/). 